Editorial

\title{
Synthesis and Properties of Bulk Nanostructured Metallic Materials
}

\author{
Byungmin Ahn
}

Department of Materials Science and Engineering and Department of Energy Systems Research, Ajou University, Suwon 16499, Korea; byungmin@ajou.ac.kr; Tel.: +82-31-219-3531

Received: 17 October 2018; Accepted: 18 October 2018; Published: 20 October 2018

check for updates

\section{Introduction and Scope}

Bulk nanostructured materials (BNMs) are defined as polycrystalline bulk solids with nanocrystalline (NC) or ultrafine-grained (UFG) microstructures. BNMs have received increasing attention because of their potentially improved properties and promising applications compared to conventional coarse-grained (CG) materials with the same chemical compositions. For example, BNMs exhibit superior mechanical properties, such as strength or hardness, unachievable with conventional counterparts. This is generally attributed to a large portion of volume being associated with disordered grain boundary regions because of extremely fine grain sizes of BNMs. Recent advances in synthesis and processing techniques of BNMs drive our need to understand underlying fundamental phenomena, as well as their significant properties. Research on synthesis and properties of BNMs is one of the most emerging fields in advanced structural materials systems. This special issue covers a wide scope in the research field of BNMs.

\section{Contributions}

Eleven research articles have been published in this Special Issue of Metals. The subjects are multidisciplinary, including (1) advances in severe plastic deformation (SPD) processing and related materials, (2) nanostructured powder processing and powder metallurgy materials, (3) additive manufacturing with nanostructures, and (4) biomedical and other functional applications with nanostructures.

First, three articles deal with SPD processing and related materials. Bruder [1] studied mechanical properties of $\mathrm{ARMCO}^{\circledR}$ iron processed by different forming techniques, including cold rolling, equal channel angular pressing (ECAP), and a combination of both processes. The article presents comparisons of microstructures and mechanical properties under monotonic and cyclic loading conditions. A combination of a single ECAP pass followed by cold rolling exhibited a good compromise between mechanical properties and processing efforts, which emphasizes application potential for precursors to UFG microstructures. Zhou et al. [2] demonstrated spontaneous modification of the surface of titanium with different substrate microstructures after soaking in a physiological solution. In this research, commercial purity (CP) titanium was processed by ECAP to achieve UFG microstructure and was immersed in simulated body fluid for 60 days. Afterwards, electrochemical impedance spectroscopy was measured and surface morphology investigated. This research was the first attempt to understand how the UFG microstructure of biomedical titanium affects chemical composition changes of its surface, providing a potential method to improve bio-bonding between an implant and surrounding tissue. The article by Zhemchuzhnikova et al. [3] presents the investigation of the Portevin-Le Chatelier (PLC) effect in an ECAP Al 5024 alloy (Al-Mg-Mn-Sc-Zr) with UFG microstructure at various strain rates. To examine behavior of PLC bands, the authors performed two different techniques, local extensometry and 2D digital image correlation (DIC). The CG material 
exhibited quasi-continuous propagation of deformation bands in all strain rate ranges, while UFG grain structure refined by ECAP led to a transition to relay-race propagation at higher strain rates and static strain localization at lower strain rates.

Second, the nanostructured powder metallurgy process was discussed in two articles. Edalati et al. [4] studied effects of $\mathrm{SiC}$ nanoparticles on properties of titanium-matrix foam. In this research various amount of $\mathrm{SiC}$ nanoparticles and $\mathrm{TiH}_{2}$ foaming agent were added to $\mathrm{CP}$ titanium powder to fabricate a titanium-matrix composite foam. Results showed that the composite foam with 57-70 vol.\% porosity was obtained and energy absorption of this composite foam increased with increasing $\mathrm{SiC}$ weight percentage. Si et at. [5] discovered a bulk NC invar alloy with a high density and significantly enhanced hardness. The invar BNM was fabricated using arc discharged Fe-Ni nanoparticles and subsequent compaction and sintering. According to thermal analysis, although the compacted Fe-Ni nanoparticles grew rapidly at $956{ }^{\circ} \mathrm{C}$, oxide impurities were completely removed by following hydrogen at $735^{\circ} \mathrm{C}$, which was optimum for synthesis of oxide-free invar BNM.

Third, about additive manufacturing with nanostructures, Cho et al. [6] employed newly developed alternative ultrasonic nanocrystal surface modification (UNSM) processes to further improve mechanical properties and tribological behavior of additive manufactured steel. In this research, a plate of AISI H13 tool steel was manufactured using a directed energy deposition (DED) additive manufacturing technique. UNSM treatment was performed on the plate using a piezoelectric-actuated precise device. Results demonstrated excellent mechanical and tribological properties for UNSM treated steel, primarily attributed to UFG microstructure on the surface.

Finally, five articles performed research on nanostructured materials for various functional and biomedical applications such as batteries, electronics, medicine, and high energy density reactants. Park et al. [7] investigated a novel process that successfully recovered a nanostructured cathode from spent lithium-ion batteries and re-synthesized new cathode material. The author employed malic acid as a leaching agent and oxalic acid as a precipitating agent, resulting in the formation of nanostructured cobalt oxalate from which the new $\mathrm{LiCoO}_{2}$ cathode was synthesized. This novel process successfully demonstrated a complete recycling of cathode material from spent lithium-ion batteries. Sharma et al. [8] produced uniform and ordered nanostructured arrays of pure Sn using a chemical pulse plating method from an aqueous sulfate bath and anodic aluminum oxide (AAO). The various nanostructures (nanoplates, nanorods, and nanoparticles) were formed on account of the tip effect of Sn nuclei inside the AAO template. In view of their potential applications in recent bulk microelectronic packaging devices, an optimum deposition potential for each kind of nanostructure needs to be controlled for mass production. Joya et al. [9] studied the synthesis of $\mathrm{Nb}_{2} \mathrm{O}_{5}$ nanoparticles through both sol-gel and Pechini methods and characterized their morphology and photoluminescent properties. According to their results, $\mathrm{Nb}_{2} \mathrm{O}_{5}$ synthesized though the sol-gel method had orthorhombic symmetry and exhibited high pressure stability. Lee et al. [10] demonstrated a facile method to synthesize enhanced pure phase and monodisperse magnetite nanoparticles and nanocubes. The high reduction potential capability of alkaline metal reagents in the reductive environment allowed formation of a pure magnetite phase. Oh et al. [11] employed a cryomilling technique to achieve a NC lamellar structure of high energy density $\mathrm{Al}-\mathrm{Ni}$ powders. In this research, $\mathrm{Al}$ and Ni powders with a molar ratio of 50:50 were milled in liquid nitrogen to generate nanoscale lamellar structure between $\mathrm{Al}$ and Ni without forming intermetallic compounds (IMCs). This metastable interface between $\mathrm{Al}$ and $\mathrm{Ni}$ having a repeat layer structure improved reactivity at a lower exothermic reaction temperature.

\section{Conclusions and Outlook}

A variety of topics have composed this Special Issue, presenting recent developments of nanostructured materials. Nevertheless, there are still many challenges to overcome in this research field and applications still need to be more widespread. As a Guest Editor, I hope all scientific results in this Special Issue contribute to the advancement and future developments of research on nanostructured materials. 
Finally, I would like to thank all reviewers for their invaluable efforts to improve the academic quality of published research in this special issue. I would also like to give special thanks to all staff at the Metals Editorial Office, especially to Toliver Guo, Assistant Editor, who managed and facilitated the publication process.

Conflicts of Interest: The author declares no conflicts of interest.

\section{References}

1. Bruder, E. Mechanical properties of $A R M C O^{\circledR}$ iron after large and severe plastic deformation-application potential for precursors to ultrafine grained microstructures. Metals 2018, 8, 191. [CrossRef]

2. Zhou, Q.; Wang, L.; Zou, C.-H. Enhanced surface precipitates on ultrafine-grained titanium in physiological solution. Metals 2017, 7, 245. [CrossRef]

3. Zhemchuzhnikova, D.; Lebyodkin, M.; Lebedkina, T.; Mogucheva, A.; Yuzbekova, D.; Kaibyshev, R. Peculiar spatiotemporal behavior of unstable plastic flow in an AlMgMnScZr alloy with coarse and ultrafine grains. Metals 2017, 7, 325. [CrossRef]

4. Edalati, E.; Sajjadi, S.A.; Babakhani, A. Effects of SiC nanoparticles on the properties of titanium-matrix foams processed by powder metallurgy. Metals 2017, 7, 296. [CrossRef]

5. Si, P.-Z.; Choi, C.-J. High hardness nanocrystalline invar alloys prepared from Fe-Ni nanoparticles. Metals 2018, 8, 28. [CrossRef]

6. Cho, I.-S.; Lee, C.-S.; Choi, C.-H.; Lee, H.-G.; Lee, M.G.; Jeon, Y. Effect of the ultrasonic nanocrystalline surface modification (UNSM) on bulk and 3D-printed AISI H13 tool steels. Metals 2017, 7, 510. [CrossRef]

7. Park, Y.M.; Lim, H.; Moon, J.-H.; Lee, H.-N.; Son, S.H.; Kim, H.; Kim, H.-J. High-yield one-pot recovery and characterization of nanostructured cobalt oxalate from spent lithium-ion batteries and successive re-synthesis of $\mathrm{LiCoO}_{2}$. Metals 2017, 7, 303. [CrossRef]

8. Sharma, A.; Srivastava, A.K.; Jeon, Y.; Ahn, B. Template-assisted fabrication of nanostructured tin ( $\beta$-Sn) arrays for bulk microelectronic packaging devices. Metals 2018, 8, 347. [CrossRef]

9. Joya, M.R.; Barba Ortega, J.J.; Raba Parez, A.M.; de Silva Filho, J.G.; Freire, P.T.C. Synthesis and characterization of nano-particles of niobium pentoxide with orthorhombic symmetry. Metals 2017, 7, 142. [CrossRef]

10. Lee, K.; Lee, S.; Oh, M.C.; Ahn, B. Alkaline metal reagent-assisted synthesis of monodisperse iron oxide nanostructures. Metals 2018, 8, 107. [CrossRef]

11. Oh, M.; Oh, M.C.; Han, D.; Jung, S.-H.; Ahn, B. Exothermic reaction kinetics in high energy density Al-Ni with nanoscale multilayers synthesized by cryomilling. Metals 2018, 8, 121. [CrossRef]

(C) 2018 by the author. Licensee MDPI, Basel, Switzerland. This article is an open access article distributed under the terms and conditions of the Creative Commons Attribution (CC BY) license (http://creativecommons.org/licenses/by/4.0/). 